\title{
Critical illness polyneuropathy and myopathy in the intensive care unit
}

\author{
Wolfgang Zink, Rainer Kollmar and Stefan Schwab
}

\begin{abstract}
Critical illness polyneuropathy (CIP) and critical illness myopathy (CIM) are major complications that occur in severely ill patients who require intensive care treatment. CIP and CIM affect the limb and respiratory muscles, and, as a consequence, they characteristically complicate weaning from the ventilator, increase the length of stay on the intensive care unit, and prolong physical rehabilitation. The basic pathophysiology of both disorders is complex and involves metabolic, inflammatory and bioenergetic alterations. It is unclear at present whether CIP and CIM are distinct entities, or whether they just represent different 'organ' manifestations of a common pathophysiological mechanism. This article provides an overview of the clinical and diagnostic features of CIP and CIM and discusses current pathophysiological and therapeutic concepts relating to these neuromuscular disorders.
\end{abstract}

Zink, W. et al. Nat. Rev. Neurol. 5, 372-379 (2009); doi:10.1038/nrneurol.2009.75

\section{Introduction}

As a result of improvements in the survival of critically ill patients over the past few decades, acquired muscular weakness - a severe and costly medical complication-is seen increasingly often on intensive care units (ICUs). ${ }^{1}$ Muscular weakness is not a 'new' phenomenon in critical illness (by definition, a disease or state in which death is possible or imminent), and was described by Osler as early as $1892 .^{2}$ At that time, the observed 'rapid loss of flesh' and respiratory muscle weakness that occurred in cases of severe sepsis were attributed to catabolic myopathy and diaphragmatic fatigue, respectively. More than 50 years later, Mertens and colleagues characterized 'coma-polyneuropathies' due to circulatory shock, and they suggested metabolic and ischemic lesions of peripheral nerves as basic pathophysiological mechanisms that could underlie these phenomena. ${ }^{3}$

In 1984, Bolton and co-workers initiated a series of studies with the aim of defining clinical, morphological and electrophysiological changes associated with acquired neuromuscular disorders, and they subsequently introduced the term 'critical illness polyneuropathy' (CIP). The investigators hypothesized that CIP, defined as a primary distal axonal degeneration of both motor and sensory fibers, was directly attributable to the 'toxic' effects of sepsis. ${ }^{4-7}$

During the past two decades, another explanation for acquired muscular weakness-'critical illness myopathy' (CIM)- has been identified and increasingly recognized..$^{7-10}$ This primary myopathy can take various morphological forms, and has been presumed to be triggered by both sepsis and other factors, including

Competing interests

The authors declare no competing interests. the extensive use of neuromuscular blocking agents and corticosteroids. ${ }^{10}$ It remains to be seen whether CIP and CIM are distinct disorders with separate pathophysiologies, or whether they merely represent different 'organ' manifestations of a common pathophysiological mechanism.

In this Review, we provide an overview of the clinical and diagnostic features of CIP and CIM. We also discuss the current pathophysiological and therapeutic concepts relating to these conditions.

\section{Incidence}

At present, the incidence of CIP and CIM is difficult to ascertain, as the rates reported in recent studies strongly depend on the nature of specific ICU subpopulations, risk factors to which these populations were exposed, the diagnostic criteria used, and the timing of diagnosis during the acute illness. ${ }^{11-15}$ Intriguingly, recent data suggest that CIP and CIM coexist-a condition that has been termed critical illness polyneuropathy and myopathy (CIPNM) - in the majority of cases., ${ }^{9,16-18}$

Current estimates indicate that $70-80 \%$ of critically ill patients develop CIP, and, presumably, a comparable percentage develops CIM. ${ }^{19-21}$ In subpopulations in which sepsis is complicated by multiple organ failure, the incidence of CIP and/or CIM could even reach $100 \% .^{21}$ Approximately two-thirds of patients with acute respiratory distress syndrome experience these neuromuscular disorders, ${ }^{22,23}$ and in unselected patients who have required mechanical ventilation for at least 4 days the incidence of CIP and CIM ranges from 25\% to 33\% on clinical evaluation, and can reach $58 \%$ on electrophysiological evaluation. ${ }^{14,24-28}$ Furthermore, $49 \%-77 \%$ of patients will acquire CIP and/or CIM when treated in an ICU for 7 days or more. ${ }^{29-31}$ 


\section{Clinical features}

Acquired, progressive muscular weakness is the leading symptom in critically ill patients with CIP and/or CIM. ${ }^{32}$ Importantly, these two disorders cannot be clearly distinguished from each other on the basis of clinical signs and symptoms alone. ${ }^{12,33} \mathrm{~A}$ failure to wean the patient from the ventilator once sedation has worn off, in the absence of any pulmonary explanation, is a common presentation of ICU-acquired myopathy and polyneuropathy. ${ }^{12,27,30,34,35}$ Consequently, these disorders are associated with an extended duration of mechanical ventilation, an increased length of stay on the ICU, and higher costs and mortality rates. After extubation, drops in oxygen saturation and hypercapnia are frequently observed in patients with CIP and/or CIM, and, as a consequence, rates of re-intubation are as twice as high as in patients without neuromuscular disorders. ${ }^{25}$

Clinically, proximal and distal muscle groups are symmetrically flaccid in CIP and CIM, and deep-tendon reflexes can be absent, although spastic quadriplegia and isolated limb weakness have been described. ${ }^{20,36,37}$ Muscle atrophy can also occur, and is frequently more severe than would be expected from the degree of immobilization alone. ${ }^{10,38,39}$ One study compared the time-course of adductor pollicis force and fatigue patterns following tetanic ulnar nerve stimulation between patients with severe sepsis and immobilized volunteers. ${ }^{38}$ No evidence was found for increased fatigability of muscles in patients with sepsis in comparison with immobilized muscles, but peripheral muscle force was markedly decreased during sepsis. Muscle weakness was, therefore, probably attributable to sepsis-induced myopathy rather than immobilization atrophy.

Unlike CIM, CIP can lead to a distal loss of sensitivity to pain, temperature and vibration, which is difficult to detect because it is often preceded by septic encephalopathy and/or the need for sedation. ${ }^{40}$ Facial muscles are relatively spared from CIP and CIM but can sometimes be involved, and ophthalmoplegia can occur in rare cases. Nevertheless, cranial nerve pathology should always guide the examiner to search for other neurological disorders, such as Guillain-Barré syndrome. ${ }^{40,41}$ Box 1 summarizes the most common neuromuscular conditions that present with generalized weakness in the ICU and should be considered in the differential diagnosis of CIP and CIM.

\section{Electrophysiological features}

From both an epidemiological and a prognostic point of view, it is important to differentiate between CIP and CIM and thereby formulate a specific diagnosis. ${ }^{12,43,44}$ Electrophysiological tests have been established for this purpose. Studies have shown that 72 hours after admission, $50 \%$ of patients had electrophysiological signs of neuromuscular dysfunction (10\% had isolated CIM, $10 \%$ had isolated CIP, and $80 \%$ had CIPNM). ${ }^{17,21,24,40,45,46}$ Despite advances in electrophysiological testing, however, the complexity of interpretation of the findings,

\section{Key points}

- Critical illness polyneuropathy (CIP) is an acute axonal sensorimotor polyneuropathy, mainly affecting the nerves in the lower limbs of critically ill patients

- Critical illness myopathy (CIM) is an acute primary myopathy, the spectrum of which ranges from pure functional impairment with normal histology to muscle atrophy and necrosis

- CIP and CIM often coexist in critically ill patients

- CIP and CIM cannot be explicitly distinguished on the basis of clinical signs alone, so specialized electrophysiological investigations or muscle biopsy are necessary to clearly differentiate between these conditions

- The pathophysiologies of CIP and CIM are complex and incompletely understood

- No specific therapeutic approach exists for CIP and CIM, although intensive insulin therapy is suggested to reduce their incidence

Box 1 | Differential diagnosis of acquired muscular weakness

The mnemonic 'MUSCLES' can help in the differential diagnosis of acquired muscular weakness ${ }^{42}$

M Medications: steroids, neuromuscular blockers (for example, pancuronium or vecuronium), zidovudine, amiodarone

U Undiagnosed neuromuscular disorder: myasthenia, Lambert-Eaton myasthenic syndrome, inflammatory myopathies, mitochondrial myopathy, acid maltase deficiency

S Spinal cord disease: ischemia, compression, trauma, vasculitis, demyelination

C Critical illness myopathy, critical illness polyneuropathy

L Loss of muscle mass: cachectic myopathy, rhabdomyolysis

E Electrolyte disorders: hypokalemia, hypophosphatemia, hypermagnesemia

S Systemic illness: porphyria, AIDS, vasculitis, paraneoplastic syndromes, toxic disorders

as well as patient discomfort during the test, still pose considerable challenges. ${ }^{1,47}$

Electroneurography is reproducible and easy to perform at the bedside. ${ }^{48} \mathrm{~A}$ motor response is induced by transcutaneous stimulation of a peripheral nerve, and compound muscle action potentials (CMAPs) from the corresponding distal muscle are recorded. To calculate motor nerve conduction velocity, stimulation at two points along the nerve is required. Sensory or mixed nerve action potentials are obtained by stimulation of a sensory or mixed nerve, respectively, with recording electrodes being placed distal or proximal to the stimulating electrode. Distal motor and sensory latencies, motor and sensory conduction velocity, amplitude (onset to negative peak) of CMAPs and nerve action potentials, and waveforms of these potentials are all measured. ${ }^{48}$

Abnormalities in motor and sensory nerve conduction point to a neuropathic process. As CIP is a primary axonal neuropathy, CMAP and nerve action potential amplitudes are characteristically reduced, whereas conduction velocity, distal motor latencies and responses to repetitive nerve stimulation are normal. ${ }^{9,16,49}$ In 


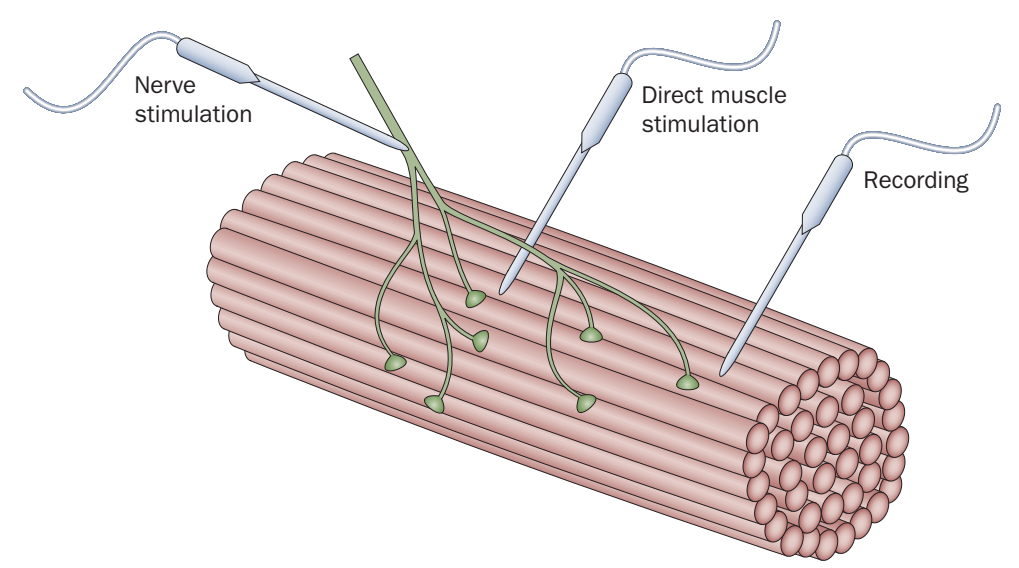

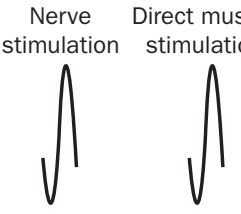

Normal

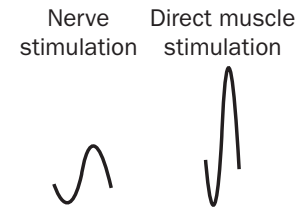

Neuropathy
Nerve Direct muscle stimulation stimulation

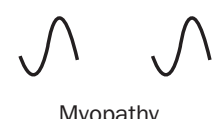

Figure 1 | Direct muscle stimulation. Stimulating and recording electrodes are both placed in the muscle so that compound muscle action potentials can be obtained even if the nerve is damaged, as in the neuropathy example shown here. In the case of myopathy, compound muscle action potentials are reduced or absent after both conventional stimulation through the nerve and direct muscle stimulation.
The third step assesses the response to maximum voluntary contraction. Increasing the force of voluntary contraction increases the firing rate of MUPs and produces systematic recruitment of additional MUPs. In healthy individuals, a large number of overlapping MUPs are recorded at maximum effort, creating a socalled interference pattern. Loss of functional motor units with axonal injury (as occurs in CIP) or conduction block produces a reduced recruitment-interference pattern. Individuals with myopathic diseases such as CIM have the normal complement of motor units but reduced numbers of functional muscle fibers, which results in a normal interference pattern with decreased amplitude. Recruitment of a large number of MUPs with weak voluntary force (early recruitment) is characteristic of a myopathic pattern.

Unfortunately, standard nerve conduction tests and electromyography often have insufficient specificity for differentiating between polyneuropathy and myopathy in critically ill patients. This difficulty can be attributed to several factors. ${ }^{52,53}$ First, CIP and CIM both lead to a reduction in the amplitude of the CMAPs, and in both conditions fibrillation potentials and/or positive sharp waves can be seen, indicating denervation or primary muscular problems. Second, the voluntary recruitment of motor units is often impossible because the muscle is too weak, or the patient is sedated or unable to cooperate for other reasons. Last, the use of SNAP alterations to discriminate neuropathies from myopathies can be problematic because many patients have peripheral edema, as discussed above.

To overcome these problems and to clearly differentiate between CIP and CIM, the concept of 'direct muscle stimulation' has recently been introduced into clinical practice (Figure 1)..$^{52,54-57}$ In this technique, which can be applied in sedated patients at the bedside, CMAPs are measured after nerve stimulation (neCMAPs) and direct muscle stimulation (dmCMAPs). By analyzing the amplitudes of the two responses, a mathematical ratio between neCMAP and dmCMAP can be calculated. An neCMAP:dmCMAP ratio $\geq 1$ suggests either myopathy or a normal clinical picture. Differentiation between $\mathrm{CIM}$ and normal muscle function can easily be accomplished on the basis of the absolute value of the dmCMAP amplitude-myopathy causes a loss of electrically excitable muscle, leading to a reduction in the dmCMAP amplitude. A neCMAP:dmCMAP ratio $<1$ indicates the presence of neuropathy. In patients with CIPNM, ratios $>0.5$ are characteristically observed. In such cases, dmCMAPs are depressed, and the reduction of neCMAPs depends on the extent to which axons are affected..$^{52}$

Despite this promising approach, CIM still cannot be detected in all cases. In pure proteolytic or necrotic forms of this condition, for example, the remaining muscle fibers can present normal membrane excitability. ${ }^{55,56}$ Furthermore, direct muscle stimulation is technically demanding and has been used only in a few centers to date. 


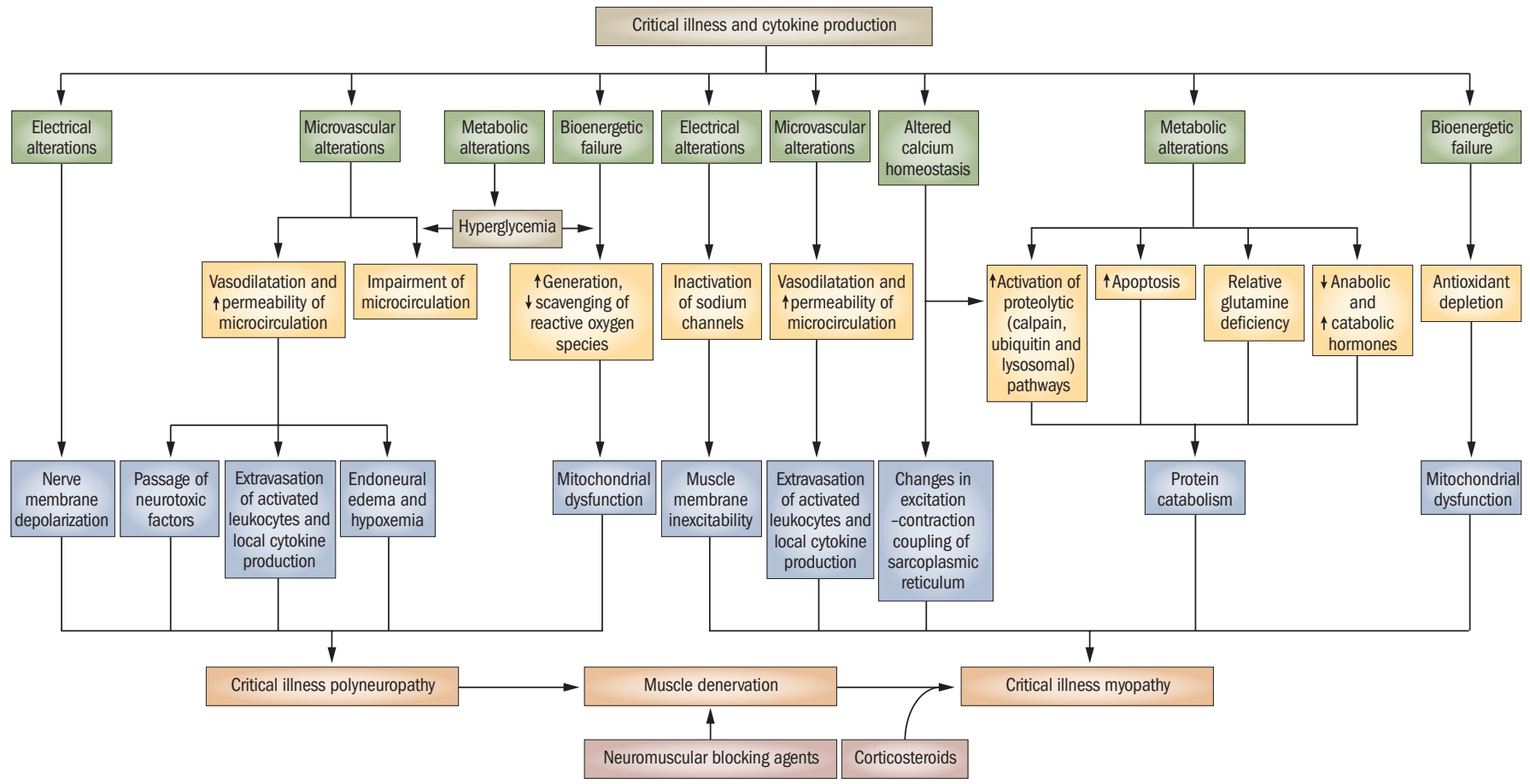

Figure 2 | Pathophysiological mechanisms of acquired muscular weakness. The network of proposed pathophysiological mechanisms involved in the development of critical illness polyneuropathy and myopathy is shown. Permission obtained from Biomed Central Ltd (c) Hermans, G. et al. Crit. Care 12, 238-246 (2008).

\section{Histopathological features}

For definitive diagnosis of CIM, muscle biopsies are considered to be the gold standard..$^{10,12,43}$ However, this procedure is highly invasive, and cannot, therefore, be applied to all patients, especially considering the high incidence of CIM.

Three subtypes of ICU-acquired myopathies have been morphologically described that are often grouped together as acute quadriplegic myopathy. ${ }^{58-63}$ Thick filament myopathy is the most common form of ICU-acquired myopathy, and is characterized by selective proteolysis and loss of myosin filaments. In minimal change or cachectic myopathy, the muscle fibers show caliber variations, angulations, internalized nuclei, rimmed vacuoles, fatty degeneration, and fibrosis. Necrotizing myopathy is characterized by muscle fiber vacuolization and phagocytosis of myocytes. The question of whether apoptotic myopathy is an independent histopathological subtype of CIM remains unanswered..$^{10}$

In patients with CIP, histopathological studies have detected morphological signs of axonal degeneration in both motor and sensory nerve fibers, resulting in extensive denervation atrophy of limb and respiratory muscles. ${ }^{79}$ Furthermore, angular atrophy of isolated scattered muscle fibers has been observed as part of an acute denervation process. ${ }^{7,9,18,36,64}$

\section{Risk factors}

Numerous studies have been carried out to attempt to identify critically ill patients who are at risk for CIM and CIP. ${ }^{32,34,65-69}$ These trials uniformly concluded that sepsis, systemic inflammatory response syndrome, and multiorgan failure are crucial premises for the occurrence of both of these neuromuscular disorders. Various additional factors have been identified as independent risk factors for CIP and CIM. These factors include female sex,${ }^{14}$ severe illness, ${ }^{24,67}$ long duration of organ dysfunction, ${ }^{14}$ renal failure and renal replacement therapy ${ }^{70}$ hyperosmolarity, ${ }^{70}$ parenteral nutrition, ${ }^{70}$ low serum albumin, ${ }^{19}$ an extended ICU stay, ${ }^{19,29}$ vasopressor and catecholamine support, ${ }^{29}$ and central neurological failure (septic encephalopathy). ${ }^{70}$ Hyperglycemia also has been identified as an independent risk factor for acquired muscular weakness, with important potential implications in terms of prevention..$^{29-31,34,71}$

The effects of long-term application of neuromuscular blocking agents and corticosteroids on neuromuscular function have been discussed in light of controversial results from a number of prospective studies. ${ }^{12,14,24,67,72}$ However, CIM has been reported to occur in patients who are receiving only one or neither of these agents, so use of these drugs is not considered to be a prerequisite for the development of CIM, CIP or both.

\section{Pathophysiology}

The various pathophysiological mechanisms that have been proposed to underlie the development of CIP and CIM are outlined in Figure 2.

The pathophysiology of CIP is complex and still not understood in detail. ${ }^{73,74}$ Sepsis-related alterations in the 
microcirculation of peripheral nerves, probably mediated by enhanced expression of E-selectin in the vascular endothelium, were suggested to be crucial events. ${ }^{64}$ Furthermore, cytokines might alter vasoregulation and increase microvascular permeability. As a result, endoneural edema progressively develops and induces hypoxemia and energy depletion; for example, by increasing intercapillary distance. Consequently, primary axonal degeneration might occur as a result of a severe deficit in energy supply. Hyperglycemia and the ensuing increased passive uptake of glucose might also contribute to neural bioenergetic failure, causing increased generation and deficient scavenging of reactive oxygen species (ROS).

Leakage of capillaries and enhanced permeability have been suggested to facilitate the passage of neurotoxic factors into the endoneurium. In addition, endothelial cell-leukocyte adhesion and extravasation of activated leukocytes are promoted by the septic state, with local cytokine production occurring in the endoneural space. In this context, direct toxic effects of cytokines on peripheral nerves as well as the existence of a specific 'neurotoxic factor' have been postulated, both of which could alter the excitability of axonal membranes..$^{50}$

The pathophysiology of CIM has not yet been completely elucidated. ${ }^{73,75}$ Muscle contraction is the result of numerous cellular and subcellular events, ranging from membrane excitation, intracellular calcium release and subsequent rises in myoplasmic calcium to the initiation of ATP-dependent cross-bridge cycling. ${ }^{10}$ Consequently, impairment of this cascade at various steps produces muscle weakness as a common symptom. Recent data unanimously point towards the idea that metabolic, inflammatory and bioenergetic alterations, as well as protein catabolism and muscle wasting, are involved in the pathogenesis of CIM. ${ }^{10}$

The probable 'physiological' aim of muscular protein breakdown during sepsis is to supply the increased need for amino acids (especially glutamine); for example, for gluconeogenesis and the synthesis of acute-phase proteins in the liver. Proteolytic pathways, such as the ubiquitin-proteasome, calpain and lysosomal pathways, are activated by proinflammatory cytokines (for example, tumor necrosis factor [TNF], interferon $\gamma$ [IFN- $\gamma]$, interleukin [IL]-1 and IL-6) and an increased rate of apoptosis. ${ }^{76-79}$ This protein degradation selectively targets the myosin heavy chains that constitute $\sim 40 \%$ of the myofibrillar protein content of adult muscle. ${ }^{10}$ Intriguingly, other myofibril proteins, such as troponin $\mathrm{T}$, tropomyosin and actin, remain unaffected. The ATP-dependent ubiquitin-proteasome system was formerly considered to be the primary cause of myofibril breakdown. ${ }^{80-82}$ Recent data show, however, that intact myofibrils cannot be degraded by this pathway, so calcium-dependent proteases such as calpain are now suggested to have a predominant role in the initial phase of protein breakdown. In a second step, proteins are further processed by the proteasome. Decreased levels of anabolic hormones and increased levels of catabolic hormones might contribute to myofilament loss and apoptosis in this situation. ${ }^{1}$ TNF and IFN- $\gamma$ inhibit intrinsic repair mechanisms through suppression of the MYOD1 gene, which is essential for the differentiation of newly formed myotubes..$^{83}$ As a result, total amino acid concentrations-especially glutamine levels-decrease within skeletal muscle tissue. ${ }^{37}$

Another potential mechanism in the pathogenesis of CIM is an acquired channelopathy, which frequently results in hypoexcitability or inexcitability of muscle cell membranes. ${ }^{84-87}$ In the first phase of sepsis, high levels of proinflammatory cytokines stimulate the hypothalamus-pituitary axis, thereby temporarily increasing endogenous corticosteroid concentrations. However, adrenal insufficiency can subsequently develop, thereby causing the cytokine:corticosteroid ratio to increase as a result of a drop in corticosteroid levels. ${ }^{10}$ Inactivation of sodium channels at the resting potential and a shift in the voltage dependence of channel inactivation are suggested to result from these sepsis-related events. ${ }^{85}$ Furthermore, impaired expression of nitric oxide synthetases could contribute to reduced muscle membrane excitability, as nitric oxide is involved in maintaining the resting potential of myocytes ${ }^{88}$ Recent experimental data have shown that alterations in the excitation-contraction coupling process, as well as in the regulation of intracellular calcium homeostasis by the sarcoplasmic reticulum, are also involved in the pathogenesis of CIM. ${ }^{89}$ The existence of a myotoxic serum factor, which would have additional effects on the excitability of muscle fiber membranes, has been postulated..$^{90}$

Further mechanisms that are suggested to contribute to the pathogenesis of CIM are bioenergetic and metabolic breakdowns. ${ }^{1}$ As mentioned above, hepatic gluconeogenesis is increased during sepsis despite hyperglycemia and elevated insulin levels. ${ }^{73}$ This phenomenon can be explained by a relative hepatomuscular insulin resistance, which results in impaired mitochondrial function and ATP depletion, thereby creating 'cytopathic hypoxia' in the myocytes. ${ }^{40,91,92}$ Activation of the ubiquitin-proteasome pathway and induction of nitric oxide synthetase further decrease intracellular ATP levels in this situation. In turn, nitric oxide overproduction inhibits the respiratory chain (especially complexes I and IV), thereby increasing mitochondrial release of ROS. As a consequence, intracellular levels of antioxidants-especially glutathione-decrease, and levels of ROS rise, with a disastrous impact on the structural integrity of the myocytes. In addition, mitochondrial release of cytochrome $\mathrm{C}$ could act as a proapoptotic signal. ${ }^{73,93}$

Good evidence exists that immune mechanisms are also involved in the pathogenesis of CIM, as activated leukocytes producing proinflammatory and antiinflammatory cytokines infiltrate the skeletal muscle during sepsis. ${ }^{18}$

The exact roles of corticosteroids and NMBAs in CIM are not clearly understood. ${ }^{23,94}$ Experimental data show 
that muscle damage can be attenuated by administration of specific muscle corticosteroid receptor antagonists, which indicates that sepsis-induced muscle changes are mediated, at least partly, by endogenous corticosteroids. ${ }^{77}$ Long-term application of nondepolarizing NMBAs is suggested to cause muscular weakness by upregulating juvenile acetylcholine receptors (which have lower acetylcholine sensitivity than the adult receptors) and by impairing neurotransmitter release. ${ }^{87}$ Furthermore, NMBAs increase the quantity of myoplasmic corticosteroid receptors, thereby sensitizing muscle cells to the effects of catabolic corticosteroids. ${ }^{73,87}$ Another theory is that increased capillary permeability allows NMBAs to cross the muscle membrane and exert direct toxic effects on muscle cells or cause functional denervation of muscle. ${ }^{7}$

Functional denervation could represent the pathogenetic link between CIP and CIM. ${ }^{12}$ Muscle damage has been shown to be markedly enhanced if limbs are denervated before exposure to corticosteroids. It remains to be established whether CIP and CIM represent different manifestations of a single pathophysiological mechanism in which sepsis triggers membrane inexcitability of nerves, skeletal muscles and other tissue types. ${ }^{50,95}$

\section{Preventive and therapeutic approaches}

On the basis of pathogenetic considerations, several therapeutic strategies have been proposed for the prevention of CIP and CIM. ${ }^{1,10,12}$ These strategies include nutritional interventions, supplement and antioxidant therapy (for example, substitution of glutamine, arginine, nucleotides or omega-3 fatty acids), and the application of testosterone derivates, growth hormones and immunoglobulins. ${ }^{12,30,31,34,70}$ None of these approaches has actually been shown to have beneficial effects on muscle function, however, so there is, as yet, no specific therapy for the treatment of CIM and CIP. Consequently, only preventive and supportive measures can currently be recommended. ${ }^{29}$ The aggressive treatment of sepsis-the most important risk factor-has highest priority in terms of reducing the incidence of CIM and CIP. Furthermore, NMBAs and corticosteroids should be used at minimal doses for as short a period as possible, although 'stress doses' of steroids ( $<250 \mathrm{mg}$ per day) are not suggested to induce CIM. ${ }^{10}$

The effects of intensive versus conventional insulin therapy have been prospectively studied in surgical and medical ICU patients. ${ }^{30,31,34}$ Subanalyses showed that intensive insulin therapy reduced the collective incidence of CIM and CIP from $49 \%$ to $25 \%$ in surgical ICU patients, and from $51 \%$ to $39 \%$ in medical ICU patients. Furthermore, the rate of extended mechanical ventilation ( $>2$ weeks) was markedly reduced in both settings. Multivariate analysis unequivocally attributed the decreased incidence of CIM and CIP to intensive insulin therapy (aiming at glycemia of $80-110 \mathrm{mg} / \mathrm{dl}$ [4.440-6.105 mmol/l] ). ${ }^{29}$ Concerns have been raised, however, regarding safety, risk of hypoglycemia, and limitations of the diagnostic methods used in these trials, and these results have not yet been confirmed in a larger population.

There are some indications that early mobilization and physiotherapy could improve outcomes in patients with CIP or CIM, but this possibility is still open to debate. ${ }^{96,97}$

\section{Prognosis}

CIM and CIP both have a strong bearing on outcomes for critically ill patients, as both disorders are associated with an increased length of stay on the ICU and in hospital overall, and with elevated mortality rates. ${ }^{1}$ Spontaneous recovery occurs within weeks in mild cases and within months in severe cases, but can be incomplete or not occur at all in some patients. ${ }^{98,99}$ Complete recovery has been reported in over $50 \%$ of patients with CIP and/or CIM, although rehabilitation is markedly impaired after ICU treatment for 4 weeks or more..$^{28,100}$ Unrecordable CMAPs have been postulated to be a predictor of sustained functional disability, and clinical and neurophysiological signs can be present for up to 5 years. ${ }^{100}$ Among the most severe cases, approximately one-third of surviving patients are thought to remain severely disabled with tetraparesis, tetraplegia or paraplegia, and to experience substantial impairment of quality of life. ${ }^{98}$

One issue that remains controversial is the question of whether the differentiation between CIP and CIM has any influence on the prognosis for patients in an ICU. The results of an Italian, 1-year, multicenter prospective cohort study of patients with CIM, CIP or CIPNM were published in 2008. ${ }^{13}$ In this study, known as CRIMYNE (CRitical Illness MYopathy and/or Neuropathy), the outcomes in 28 patients suggested that CIM has a better prognosis than CIP, given that out of four CIP patients followed for 1 year, only one recovered, whereas five out of six patients with CIM recovered completely within 6 months. The number of patients was, however, too small for definite conclusions to be drawn.

\section{Conclusions}

Acquired muscular weakness due to CIM and CIP is a severe and costly complication, the incidence of which continues to increase in ICUs. Characteristically, CIP and CIM delay weaning from the ventilator, compromise rehabilitation, and are associated with increased hospital and ICU stays and elevated mortality rates. Clinical examination, electrophysiological tests and-in rare cases-nerve and muscle biopsies are required to diagnose these neuromuscular disorders, and to clearly differentiate CIP from CIM. Experimental and clinical studies suggest complex and multifactorial pathophysiologies for both disorders.

Until recently, no therapeutic measures had been shown to be effective in lowering the incidence of CIP and CIM. Interestingly, however, intensive insulin therapy seems 
to significantly reduce the electrophysiological incidence of these conditions, as well as the duration of mechanical ventilation and 180-day mortality, in patients in both surgical and medical ICUs. ${ }^{34}$ Nevertheless, concerns have been raised over the general safety of this approach and the risk of hypoglycemia, as well as limitations of the diagnostic methods used in these trials. Future studies must aim to define strict diagnostic criteria for CIP and CIM, and to evaluate a range of interventions to prevent acquired polyneuropathy and myopathy in patients who are critically ill.

\section{Review criteria}

PubMed was searched for articles published before 15 April 2009 , including electronic early release publications. Search terms included "critical illness myopathy", "critical illness polyneuropathy", "sepsis and skeletal muscle", "sepsis and nerve", and "acquired muscular weakness and critically ill". We chose only papers written in English and German, the languages read by the authors. In addition, we searched the clinical trials registries http://clinicaltrials.gov/ and http:// www.controlled-trials.com/mrct/ with the terms "critical illness myopathy" and "critical illness polyneuropathy".
1. Latronico, N. \& Guarneri, B. Critical illness myopathy and neuropathy. Minerva Anestesiol. 74, 319-323 (2008).

2. Osler, W. The Principles and Practice of Medicine, 1st edn (D. Appleton, New York, 1892).

3. Mertens, H. G. Disseminated neuropathy following coma. On the differentation of socalled toxic neuropathy [German]. Nervenarzt 32, 71-79 (1961).

4. Bolton, C. F., Gilbert, J. J., Hahn, A. F. \& Sibbald, W. J. Polyneuropathy in critically ill patients. J. Neurol. Neurosurg. Psychiatry 47, 1223-1231 (1984).

5. Bolton, C. F. et al. Critically ill polyneuropathy: electrophysiological studies and differentiation from Guillain-Barré syndrome. J. Neurol. Neurosurg. Psychiatry 49, 563-573 (1986).

6. Bolton, C. F. Sepsis and the systemic inflammatory response syndrome: neuromuscular manifestations. Crit. Care Med. 24, 1408-1416 (1996).

7. Bolton, C. F. Neuromuscular manifestations of critical illness. Muscle Nerve 32, 140-163 (2005).

8. Lacomis, D., Giuliani, M. J., Van Cott, A. \& Kramer, D. J. Acute myopathy of intensive care: clinical, electromyographic, and pathological aspects. Ann. Neurol. 40, 645-654 (1996).

9. Latronico, N. et al. Critical illness myopathy and neuropathy. Lancet 347, 1579-1582 (1996).

10. Friedrich, O. Critical illness myopathy: sepsismediated failure of the peripheral nervous system. Eur. J. Anaesthesiol. Suppl. 42, 73-82 (2008).

11. Stevens, R. D. et al. Neuromuscular dysfunction acquired in critical illness: a systematic review. Intensive Care Med. 33, 1876-1891 (2007).

12. Hermans, G., De Jonghe, B., Bruyninckx, F. $\&$ Van den Berghe, G. Clinical review: critical illness polyneuropathy and myopathy. Crit. Care 12, 238 (2008).

13. Guarneri, B., Bertolini, G. \& Latronico, N. Long term outcome in patients with critical illness myopathy or neuropathy: the Italian multicentre CRIMYNE study. J. Neurol. Neurosurg. Psychiatry 79, 838-841 (2008).

14. De Jonghe, B. et al. Paresis acquired in the intensive care unit: a prospective multicenter study. JAMA 288, 2859-2867 (2002).

15. Ali, N. A. et al. Acquired weakness, handgrip strength, and mortality in critically ill patients. Am. J. Respir. Crit. Care Med. 178, 261-268 (2008).

16. Bednarik, J., Lukas, Z. \& Vondracek, P. Critical illness polyneuromyopathy: the electrophysiological components of a complex entity. Intensive Care Med. 29, 1505-1514 (2003).

17. Khan, J., Harrison, T. B., Rich, M. M. \& Moss, M. Early development of critical illness myopathy and neuropathy in patients with severe sepsis. Neurology 67, 1421-1425 (2006).

18. De Letter, M. A et al. Critical illness polyneuropathy and myopathy (CIPNM): evidence for local immune activation by cytokineexpression in the muscle tissue. J. Neuroimmunol. 106, 206-213 (2000).

19. Witt, N. J. et al. Peripheral nerve function in sepsis and multiple organ failure. Chest 99 , 176-184 (1991).

20. Hund, E. Neurological complications of sepsis: critical illness polyneuropathy and myopathy. J. Neurol. 248, 929-934 (2001).

21. Tennila, A. et al. Early signs of critical illness polyneuropathy in ICU patients with systemic inflammatory response syndrome or sepsis. Intensive Care Med. 26, 1360-1363 (2000).

22. Bercker, S. et al. Critical illness polyneuropathy and myopathy in patients with acute respiratory distress syndrome. Crit. Care Med. 33, 711-715 (2005).

23. Steinberg, K. P. et al. Efficacy and safety of corticosteroids for persistent acute respiratory distress syndrome. N. Engl. J. Med. 354, 1671-1684 (2006).

24. de Letter, M. A. et al. Risk factors for the development of polyneuropathy and myopathy in critically ill patients. Crit. Care Med. 29 , 2281-2286 (2001)

25. De Jonghe, B., Bastuji-Garin, S., Sharshar, T. Outin, H. \& Brochard, L. Does ICU-acquired paresis lengthen weaning from mechanical ventilation? Intensive Care Med. 30, 1117-1121 (2004).

26. Leijten, F. S. et al. Critical illness polyneuropathy in multiple organ dysfunction syndrome and weaning from the ventilator. Intensive Care Med. 22, 856-861 (1996).

27. Garnacho-Montero, J., Amaya-Villar, R., Garcia-Garmendia, J. L., Madrazo-Osuna, J. \& Ortiz-Leyba, C. Effect of critical illness polyneuropathy on the withdrawal from mechanical ventilation and the length of stay in septic patients. Crit. Care Med. 33, 349-354 (2005).

28. Leijten, F. S., Harinck-de Weerd, J. E. Poortvliet, D. C. \& de Weerd, A. W. The role of polyneuropathy in motor convalescence after prolonged mechanical ventilation. JAMA 274 1221-1225 (1995)

29. Van den Berghe, G., Schoonheydt, K., Becx, P., Bruyninckx, F. \& Wouters, P. J. Insulin therapy protects the central and peripheral nervous system of intensive care patients. Neurology 64 , 1348-1353 (2005).

30. Hermans, G. et al. Benefits of intensive insulin therapy on neuromuscular complications in routine daily critical care practice: a retrospective study. Crit. Care 13, R5 (2009).
31. Hermans, G. et al. Impact of intensive insulin therapy on neuromuscular complications and ventilator dependency in the medical intensive care unit. Am. J. Respir. Crit. Care Med. 175, 480-489 (2007).

32. Schweickert, W. D. \& Hall, J. ICU-acquired weakness. Chest 131, 1541-1549 (2007).

33. Lacomis, D. \& Campellone, J. V. Critical illness neuromyopathies. Adv. Neurol. 88, 325-335 (2002).

34. Hermans, G., De Jonghe, B., Bruyninckx, F. \& Van den Berghe, G. Interventions for preventing critical illness polyneuropathy and critical illness myopathy. Cochrane Database of Systematic Reviews, Issue 1. Art. No.: CD006832. doi:10.1002/14651858.CD006832.pub2 (2009).

35. De Jonghe, B. et al. Respiratory weakness is associated with limb weakness and delayed weaning in critical illness. Crit. Care Med. 35, 2007-2015 (2007).

36. Zochodne, D. W. et al. Critical illness polyneuropathy. A complication of sepsis and multiple organ failure. Brain 110, 819-841 (1987).

37. Burnham, E. L., Moss, M. \& Ziegler, T. R. Myopathies in critical illness: characterization and nutritional aspects. J. Nutr. 135, S1818-S1823 (2005).

38. Eikermann, M. et al. Muscle force and fatigue in patients with sepsis and multiorgan failure. Intensive Care Med. 32, 251-259 (2006).

39. Zifko, U. A., Zipko, H. T. \& Bolton, C. F. Clinical and electrophysiological findings in critical illness polyneuropathy. J. Neurol. Sci. 159 , 186-193 (1998).

40. Bird, S. J. Diagnosis and management of critical illness polyneuropathy and critical illness myopathy. Curr. Treat. Options Neurol. 9, 85-92 (2007).

41. van Mook, W. N. \& Hulsewe-Evers, R. P. Critical illness polyneuropathy. Curr. Opin. Crit. Care $\mathbf{8}$ 302-310 (2002)

42. Maramattom, B. V. \& Wijdicks, E. F. Acute neuromuscular weakness in the intensive care unit. Crit. Care Med. 34, 2835-2841 (2006).

43. Dhand, U. K. Clinical approach to the weak patient in the intensive care unit. Respir. Care 51, 1024-1040 (2006).

44. Schwarz, J., Planck, J., Briegel, J. \& Straube, A Single-fiber electromyography, nerve conduction studies, and conventional electromyography in patients with critical-illness polyneuropathy: evidence for a lesion of terminal motor axons. Muscle Nerve 20, 696-701 (1997).

45. Gorson, K. C. Approach to neuromuscular disorders in the intensive care unit. Neurocrit. Care 3, 195-212 (2005). 
46. Latronico, N. Neuromuscular alterations in the critically ill patient: critical illness myopathy, critical illness neuropathy, or both? Intensive Care Med. 29, 1411-1413 (2003).

47. De Jonghe, B., Lacherade, J. C., Durand, M. C. \& Sharshar, T. Critical illness neuromuscular syndromes. Crit. Care Clin. 22, 805-818 (2006).

48. Botteri, M. \& Guarneri, B. Electrophysiologica tests in intensive care. Eur. J. Anaesthesiol. Suppl. 42, 174-180 (2008).

49. Latronico, N. et al. Simplified electrophysiological evaluation of peripheral nerves in critically ill patients: the Italian multicentre CRIMYNE study. Crit. Care 11, R11 (2007).

50. Z'Graggen, W. J., Lin, C. S., Howard, R. S., Beale, R. J. \& Bostock, H. Nerve excitability changes in critical illness polyneuropathy. Brain 129, 2461-2470 (2006).

51. Z'Graggen, W. J. \& Bostock, H. Nerve membrane excitability testing. Eur. J. Anaesthesiol. Suppl. 42, 68-72 (2008).

52. Seghelini, E. Direct stimulation: a useful technique. Eur. J. Anaesthesiol. Suppl. 42, 181-185 (2008).

53. Rich, M. M., Bird, S. J., Raps, E. C., McCluskey, L. F. \& Teener, J. W. Direct muscle stimulation in acute quadriplegic myopathy. Muscle Nerve 20, 665-673 (1997).

54. Rich, M. M., Raps, E. C. \& Bird, S. J. Distinction between acute myopathy syndrome and critical illness polyneuropathy. Mayo Clin. Proc. 70, 198-200 (1995)

55. Trojaborg, W., Weimer, L. H. \& Hays, A. P. Electrophysiologic studies in critical illness associated weakness: myopathy or neuropathy-a reappraisal. Clin. Neurophysiol. 112, 1586-1593 (2001)

56. Trojaborg, W. Electrophysiologic techniques in critical illness-associated weakness. J. Neurol. Sci. 242, 83-85 (2006).

57. Lefaucheur, J. P., Nordine, T., Rodriguez, P. \& Brochard, L. Origin of ICU acquired paresis determined by direct muscle stimulation. J. Neurol. Neurosurg. Psychiatry 77, 500-506 (2006).

58. Danon, M. J. \& Carpenter, S. Myopathy with thick filament (myosin) loss following prolonged paralysis with vecuronium during steroid treatment. Muscle Nerve 14, 1131-1139 (1991).

59. Hasselgren, P. O. \& Fischer, J. E. Sepsis: stimulation of energy-dependent protein breakdown resulting in protein loss in skeletal muscle. World J. Surg. 22, 203-208 (1998).

60. Norman, $\mathrm{H}$. et al. A porcine model of acute quadriplegic myopathy: a feasibility study. Acta Anaesthesiol. Scand. 50, 1058-1067 (2006).

61. Hund, E. Myopathy in critically ill patients. Crit. Care Med. 27, 2544-2547 (1999).

62. Latronico, N., Peli, E. \& Botteri, M. Critical illness myopathy and neuropathy. Curr. Opin. Crit. Care 11, 126-132 (2005)

63. Leung, T. W. et al. Myopathic changes associated with severe acute respiratory syndrome: a postmortem case series. Arch. Neurol. 62, 1113-1117 (2005)

64. Fenzi, F., Latronico, N., Refatti, N. \& Rizzuto, N. Enhanced expression of E-selectin on the vascular endothelium of peripheral nerve in critically ill patients with neuromuscular disorders. Acta Neuropathol. 106, 75-82 (2003).

65. Young, G. B. Critical illness myopathy: deeper insights. Crit. Care Med. 36, 1977 (2008).
66. Nanas, S. et al. Predisposing factors for critical illness polyneuromyopathy in a multidisciplinary intensive care unit. Acta Neurol. Scand. 118, 175-181 (2008).

67. Bednarik, J., Vondracek, P., Dusek, L. Moravcova, E. \& Cundrle, I. Risk factors for critical illness polyneuromyopathy. J. Neurol. 252, 343-351 (2005).

68. Visser, L. H. Critical illness polyneuropathy and myopathy: clinical features, risk factors and prognosis. Eur. J. Neurol. 13, 1203-1212 (2006).

69. Hough, C. L. \& Needham, D. M. The role of future longitudinal studies in ICU survivors: understanding determinants and pathophysiology of weakness and neuromuscular dysfunction. Curr. Opin. Crit. Care 13, 489-496 (2007).

70. Garnacho-Montero, J. et al. Critical illness polyneuropathy: risk factors and clinical consequences. A cohort study in septic patients. Intensive Care Med. 27, 1288-1296 (2001).

71. Van den Berghe, G. Insulin therapy for the critically ill patient. Clin. Cornerstone 5, 56-63 (2003).

72. Herridge, M. S. et al. One-year outcomes in survivors of the acute respiratory distress syndrome. N. Engl. J. Med. 348, 683-693 (2003).

73. Friedrich, O. Critical illness myopathy: what is happening? Curr. Opin. Clin. Nutr. Metab. Care 9 403-409 (2006).

74. Khan, J., Harrison, T. B. \& Rich, M. M. Mechanisms of neuromuscular dysfunction in critical illness. Crit. Care Clin. 24, 165-177 (2008).

75. Friedrich, O., Fink, R. H. \& Hund, E. Understanding critical illness myopathy: approaching the pathomechanism. J. Nutr. 135 S1813-S1817 (2005).

76. Showalter, C. J. \& Engel, A. G. Acute quadriplegic myopathy: analysis of myosin isoforms and evidence for calpain-mediated proteolysis. Muscle Nerve 20, 316-322 (1997).

77. Tiao, G. et al. Sepsis is associated with increased mRNAs of the ubiquitin-proteasome proteolytic pathway in human skeletal muscle. J. Clin. Invest. 99, 163-168 (1997).

78. Klaude, M. et al. Proteasome proteolytic activity in skeletal muscle is increased in patients with sepsis. Clin. Sci. (Lond.) 112, 499-506 (2007).

79. Di Giovanni, S., Mirabella, M., D’Amico, A., Tonali, P. \& Servidei, S. Apoptotic features accompany acute quadriplegic myopathy. Neurology 55, 854-858 (2000).

80. Solomon, V. \& Goldberg, A. L. Importance of the ATP-ubiquitin-proteasome pathway in the degradation of soluble and myofibrillar proteins in rabbit muscle extracts. J. Biol. Chem. 271 26690-26697 (1996)

81. Solomon, V., Lecker, S. H. \& Goldberg, A. L. The $\mathrm{N}$-end rule pathway catalyzes a major fraction of the protein degradation in skeletal muscle. J. Biol. Chem. 273, 25216-25222 (1998).

82. Williams, A. B. et al. Sepsis stimulates release of myofilaments in skeletal muscle by a calciumdependent mechanism. FASEB J. 13, 1435-1443 (1999).

83. Acharyya, S. et al. Cancer cachexia is regulated by selective targeting of skeletal muscle gene products. J. Clin. Invest. 114, 370-378 (2004).

84. Rich, M. M. \& Pinter, M. J. Sodium channel inactivation in an animal model of acute quadriplegic myopathy. Ann. Neurol. 50, 26-33 (2001).

85. Rich, M. M. \& Pinter, M. J. Crucial role of sodium channel fast inactivation in muscle fibre inexcitability in a rat model of critical illness myopathy. J. Physiol. 547, 555-566 (2003).

86. Rich, M. M., Teener, J. W., Raps, E. C., Schotland, D. L. \& Bird, S. J. Muscle is electrically inexcitable in acute quadriplegic myopathy. Neurology 46, 731-736 (1996).

87. Rich, M. M., Teener, J. W., Raps, E. C. \& Bird, S. J. Muscle inexcitability in patients with reversible paralysis following steroids and neuromuscular blockade. Muscle Nerve 21, 1231-1232 (1998).

88. Capasso, M. et al. Possible role for nitric oxide dysregulation in critical illness myopathy. Muscle Nerve 37, 196-202 (2008).

89. Zink, W. et al. Alterations in intracellular $\mathrm{Ca}^{2+}$-homeostasis of skeletal muscle fibers during sepsis. Crit. Care Med. 36, 1559-1563 (2008).

90. Friedrich, O., Hund, E., Weber, C., Hacke, W. \& Fink, R. H. Critical illness myopathy serum fractions affect membrane excitability and intracellular calcium release in mammalian skeletal muscle. J. Neurol. 251, 53-65 (2004).

91. Van den Berghe, G. et al. Intensive insulin therapy in mixed medical/surgical intensive care units: benefit versus harm. Diabetes 55 , 3151-3159 (2006).

92. Van den Berghe, G. et al. Outcome benefit of intensive insulin therapy in the critically ill: Insulin dose versus glycemic control. Crit. Care Med. 31, 359-366 (2003).

93. Lydon, A. \& Martyn, J. A. Apoptosis in critical illness. Int. Anesthesiol. Clin. 41, 65-77 (2003).

94. Hough, C., Steinberg, K. P., Taylor Thompson, B., Rubenfeld, G. D. \& Hudson, L. D. Intensive care unit-acquired neuromyopathy and corticosteroids in survivors of persistent ARDS. Intensive Care Med. 35, 63-68 (2009)

95. Teener, J. W. \& Rich, M. M. Dysregulation of sodium channel gating in critical illness myopathy. J. Muscle Res. Cell Motil. 27, 291-296 (2006)

96. Morris, P. E. et al. Early intensive care unit mobility therapy in the treatment of acute respiratory failure. Crit. Care Med. 36 , 2238-2243 (2008).

97. Needham, D. M. Mobilizing patients in the intensive care unit: improving neuromuscular weakness and physical function. JAMA $\mathbf{3 0 0}$ 1685-1690 (2008).

98. de Seze, M. et al. Critical illness polyneuropathy. A 2-year follow-up study in 19 severe cases. Eur. Neurol. 43, 61-69 (2000).

99. Latronico, N., Shehu, I. \& Seghelini, E. Neuromuscular sequelae of critical illness. Curr. Opin. Crit. Care 11, 381-390 (2005).

100. Kerbaul, F. et al. Combination of histopathological and electromyographic patterns can help to evaluate functional outcome of critical ill patients with neuromuscular weakness syndromes. Crit. Care 8, R358-R366 (2004).

Acknowledgments

Rainer Kollmar and Wolfgang Zink contributed equally to this article. 